\title{
Work function performance of a C12A7 electride surface exposed to low pressure low temperature hydrogen plasmas
}

\author{
A. Heiler, ${ }^{1,2, \text { a) }}$ K. Waetzig, ${ }^{3}$ M. Tajmar, ${ }^{4}$ R. Friedl, ${ }^{2}$ R. Nocentini, ${ }^{1}$ and U. Fantz ${ }^{1,2}$ \\ ${ }^{1)}$ Max-Planck-Institut für Plasmaphysik, Boltzmannstrasse 2, D-85748 Garching, Germany \\ ${ }^{2)}$ AG Experimentelle Plasmaphysik, Universität Augsburg, D-86135 Augsburg, Germany \\ ${ }^{3)}$ Fraunhofer Institute for Ceramic Technologies and Systems IKTS, Department for Materials and Components, \\ Winterbergstrasse 28, D-01277 Dresden, Germany \\ ${ }^{4)}$ Institut für Luft- und Raumfahrttechnik, Technische Universität Dresden, D-01307 Dresden, \\ Germany
}

The calcium aluminate electride $\left[\mathrm{Ca}_{24} \mathrm{Al}_{28} \mathrm{O}_{64}\right]^{4+}\left(\mathrm{e}^{-}\right)_{4}\left(\mathrm{C} 12 \mathrm{~A} 7: \mathrm{e}^{-}\right)$is chemically and thermally stable in ambient atmosphere, offers strong electron donating capabilities and exhibits an intrinsic work function of $2.4 \mathrm{eV}$. Thus, it is an attractive material for electron emitter and low work function applications. For an implementation in negative hydrogen ion sources, where the electride could serve as converter surface for the $\mathrm{H}^{-}$production, compatibility with moderate vacuum conditions and exposure to low pressure low temperature hydrogen plasmas must be ensured. Hence, the stability and work function performance of a polycrystalline $\mathrm{C} 12 \mathrm{~A} 7: \mathrm{e}^{-}$sample are investigated under ion source relevant vacuum $\left(\sim 10^{-6} \mathrm{mbar}\right)$ and plasma conditions (electron temperature $\sim 2 \mathrm{eV}$, electron density $\sim 10^{16} \mathrm{~m}^{-3}$ ). The work function is measured via the photoelectric effect, showing that long-term hydrogen plasma exposure results in a steady-state surface work function of $2.75 \pm 0.10 \mathrm{eV}$. Since the electride is very sensitive to surface contaminations, an increase of the surface work function occurs as soon as the plasma is switched off. Vacuum heat treatment of the degraded C12A7: $\mathrm{e}^{-}$surface after plasma activation leads to a work function minimum of $2.9 \mathrm{eV}$ for temperatures in the range of $670-770 \mathrm{~K}$. Exceeding a temperature of $\sim 770 \mathrm{~K}$ leads to an increase of the $\mathrm{C} 12 \mathrm{~A} 7: \mathrm{e}^{-}$surface work function. The sample is stable during long-term hydrogen plasma exposure (up to 10 hours total plasma-on time tested) and no plasma-induced erosion is observed.

\section{INTRODUCTION}

The inorganic compound $\left[\mathrm{Ca}_{24} \mathrm{Al}_{28} \mathrm{O}_{64}\right]^{4+}\left(\mathrm{e}^{-}\right)_{4}$, known as $\mathrm{C} 12 \mathrm{~A} 7: \mathrm{e}^{-}$, is the first reported electride which is chemically and thermally stable in ambient atmosphere. ${ }^{1,2}$ These unique properties result from a rigid lattice framework of positively charged subnanometer-sized $\mathrm{Ca}-\mathrm{O}-\mathrm{Al}$ cages (internal diameter $\sim 4 \AA),{ }^{1}$ in which the anionic electrons are clathrated and protected from oxidation processes with external molecules such as $\mathrm{H}_{2} \mathrm{O}$ or $\mathrm{O}_{2}$. The encaged electrons occupy a socalled cage conduction band (CCB), ${ }^{3,4}$ which is formed due to the crystallographic cage structure and lies within the wide band gap $(\sim 7.5 \mathrm{eV})^{5}$ of the framework valence and conduction bands. It has been shown ${ }^{6}$ that for low incorporated electron concentrations, the $\mathrm{C} 12 \mathrm{~A} 7: \mathrm{e}^{-}$material is semiconducting via thermally activated polaronic cage hopping. For anionic electron densities larger than $10^{21} \mathrm{~cm}^{-3}$, the material exhibits a metal-like behavior with band conduction via quantum tunneling through the cage walls. Moreover, the carrier mobility and maximum anionic electron concentration $\left(\sim 2.3 \times 10^{21} \mathrm{~cm}^{-3}\right)^{7}$ have been found to be comparable for single-crystalline and polycrystalline $\mathrm{C} 12 \mathrm{~A} 7: \mathrm{e}^{-8},{ }^{8-10}$ but the highest electrical conductivity of up to $1500 \mathrm{~S} / \mathrm{cm}$ at $300 \mathrm{~K}$ has only been reached with single crystals so far. ${ }^{6,11,12}$

Due to the weakly bound character of the encaged electrons with a rather low Madelung energy gain, the $\mathrm{C} 12 \mathrm{~A} 7$ electride is a low work function material. Toda et al. ${ }^{5}$ have reported an intrinsic work function value of $2.4 \mathrm{eV}$ for cleaned single crystal samples under ultra-high vacuum (UHV) condi-

\footnotetext{
${ }^{a)}$ Electronic mail: adrian.heiler@ipp.mpg.de
}

tions. It is, however, well known that the C12A7: $\mathrm{e}^{-}$surface work function is a particularly sensitive parameter, since it is challenging to prepare chemically pure surfaces. ${ }^{5,7,13}$ First, it is very likely that an insulating contaminant layer exists on the surface after the fabrication process. Second, an electrondeficient layer is easily built in the vicinity of the surface due to possible reactions with external molecules, which affects the position of the Fermi level. ${ }^{14}$ Third, cage deformations or a subtle discrepancy of the stoichiometry at the surface can influence the electronic properties. As a consequence, C12A7: $\mathrm{e}^{-}$surface work functions are often measured substantially higher than $2.4 \mathrm{eV},{ }^{15-18}$ which emphasizes the crucial role of the surface preparation on the work function performance.

The intrinsic low work function of $\mathrm{C} 12 \mathrm{~A} 7: \mathrm{e}^{-}$in combination with its chemical stability and machinability has attracted widespread attention over the last years for versatile applications, e.g., as low temperature electron emitter, ${ }^{19,20}$ or as chemical reagent ${ }^{21-23}$. Further, it has gained interest in negative hydrogen ion source research ${ }^{24}$, where low work function surfaces are mandatory. ${ }^{25}$ In negative hydrogen ion sources, which are used at the front end of a variety of particle accelerators, $\mathrm{H}^{-}$ions are produced predominantly via surface conversion in low pressure low temperature hydrogen plasmas. Because this process is greatly enhanced by a low work function converter surface, ${ }^{26}$ the alkali metal Cs is typically evaporated into the vacuum chamber in order to reduce the work function of refractory metals by surface adsorption. It has been shown that with this technique, surface work functions of $\sim 2 \mathrm{eV}$ can be obtained after hydrogen plasma exposure. ${ }^{27,28}$ However, the high chemical reactivity of alkali metals and its complex redistribution dynamics due to plasma-surface interactions ${ }^{29}$ are challenging issues regarding stability and long-term relia- 
bility of such devices. Additionally, the required high consumption for a continuous and homogeneous deposition as well as contamination of high voltage components with $\mathrm{Cs}$ can be critical issues. Hence, alternative materials for the $\mathrm{H}^{-}$ converter surface are highly desirable and become an active research topic in ion source development. ${ }^{30-36}$ In this context, the $\mathrm{C} 12 \mathrm{~A} 7$ electride is seen as a promising material, since it exhibits a bulk work function which is only slightly higher than for Cs and offers a reduced complexity of handling. Moreover, additional $\mathrm{H}^{-}$production channels such as desorption of $\mathrm{H}^{-}$ions formed inside the nanocages in a hydrogen environment may also be of importance and could enhance the efficiency of a $\mathrm{C} 12 \mathrm{~A} 7: \mathrm{e}^{-}$converter surface. ${ }^{24}$ An implementation in negative hydrogen ion sources means, however, that the electride has to tolerate vacuum conditions of typically $10^{-7}-10^{-6}$ mbar. Furthermore, it has to withstand long-term hydrogen plasma exposure, where the surface is bombarded by a variety of particles such as hydrogen atoms, positive hydrogen ions with energies of a few eV, and photons with energies up to $\sim 15 \mathrm{eV}$. Thus, the work function performance and stability of a C12A7: $\mathrm{e}^{-}$surface under such conditions is examined in this paper. For the investigations, a polycrystalline $\mathrm{C} 12 \mathrm{~A} 7: \mathrm{e}^{-}$ceramic is installed at an inductively coupled plasma (ICP) experiment, where the targeted vacuum conditions are provided, long-term low pressure low temperature hydrogen plasmas can be performed and the work function of the $\mathrm{C} 12 \mathrm{~A} 7: \mathrm{e}^{-}$surface can be measured in situ via the photoelectric effect.

\section{EXPERIMENT}

\section{A. Sample fabrication}

The C12A7: $\mathrm{e}^{-}$ceramic was prepared as described by Waetzig et al. in Ref. 37. First, $\mathrm{CaCO}_{3}$ was mixed with $\mathrm{Al}_{2} \mathrm{O}_{3}$ in a 12:7 ratio in a tumbler mixer and melted in a platinum crucible at a temperature of $1723 \mathrm{~K}$. The melt was quickly solidified on a brass block and the cracked glass was milled with a rotary disk mill. Additionally, the C12A7 powder was milled in a ball mill with ethyl alcohol. After milling, organic binder was added to the slurry followed by a drying step. The powder was shaped by uniaxial pressing with a diameter of $30 \mathrm{~mm}$ and a height of $4 \mathrm{~mm}$. After isostatic pressing, the disk was debinded by heating in a muffle furnace to a temperature of $1073 \mathrm{~K}$ and sintered under nitrogen at $1593 \mathrm{~K}$ for $10 \mathrm{~h}$. The reduced $\mathrm{C} 12 \mathrm{~A} 7: \mathrm{e}^{-}$disk was ground by wet grinding with silicon oil to the final dimension with a diameter of $25 \mathrm{~mm}$ and a thickness of $2 \mathrm{~mm}$. The electronic state was characterized on a sample produced in the same way by measuring the specific resistance $(7.1 \mathrm{~S} / \mathrm{cm})$ with a silver-based braze as well as oxidizing the sample at a temperature of $1273 \mathrm{~K}$ in air and calculating the electron concentration $\left(1.0 \times 10^{21} \mathrm{~cm}^{-3}\right)$ by the mass increase.

\section{B. Plasma device}

The C12A7: $\mathrm{e}^{-}$sample is tested at a well characterized plasma experiment, ${ }^{38}$ which is schematically depicted in Fig. 1. It consists of a cylindrical vacuum vessel with $15 \mathrm{~cm}$ in diameter and $10 \mathrm{~cm}$ height, manufactured of stainless steel and equipped with several ports for peripheral components. An attached pumping system comprising a turbomolecular pump and rotary vane pump provides a vacuum level on the order of $10^{-6}$ mbar, limited by the use of Viton seals at the experiment. Hydrogen gas is fed to the chamber via calibrated mass flow controllers, and the background and working gas pressure are measured with a cold cathode gauge and a capacitive pressure gauge, respectively. Moreover, a differentially pumped residual gas analyzer (RGA) is installed at the experiment in order to monitor the background gases. A planar copper solenoid is located on top of the vessel and separated from it by a Borosilicate glass plate and a grounded Faraday screen. The solenoid is connected to a $27.12 \mathrm{MHz}$ radio frequency (RF) generator (max. $600 \mathrm{~W}$ output power) via an impedance matching network, so that plasmas can be generated via inductive RF coupling. Local plasma parameters, such as the electron density $n_{\mathrm{e}}$ and electron temperature $T_{\mathrm{e}}$, are determined by a movable Langmuir probe, and parameters like the gas temperature and the atomic hydrogen density are evaluated volume averaged using optical emission spectroscopy (OES). ${ }^{38,39}$ Additionally, stable and reproducible temperature conditions are assured by an installed water cooling system around the discharge vessel.

In order to place the $\mathrm{C} 12 \mathrm{~A} 7: \mathrm{e}^{-}$sample inside the vacuum chamber, a sample holder is installed at the bottom plate close to the vessel center. The holder, which is made of stainless steel, is electrically and thermally insulated from the grounded vessel walls and can be heated with a proportionalintegral-derivative (PID) controller up to a maximum temperature of $\sim 1250 \mathrm{~K}$. The temperatures both of the sample holder ( $\left.T_{\text {holder }}\right)$ and of the sample surface $\left(T_{\text {surf }}\right)$ are monitored by Ktype thermocouples.

\section{Work function diagnostic}

The work function of the sample surface, i.e., the minimum energy required to remove an electron from the interior of the solid into vacuum, is accessed in situ and non-invasively via the photoelectric effect. To this end, the broadband radiation of a $100 \mathrm{~W}$ high pressure mercury lamp in combination with interference filters is used. As illustrated in Fig. 1, the light is focused via two quartz lenses, enters the vessel through a quartz window, and illuminates a spot in the center of the sample surface. In order to select the photon energy, a motorized filter wheel is mounted in front of the mercury lamp, which can house a set of six interference filters. In total, 20 different interference filters with central wavelengths in the range of $239-852 \mathrm{~nm}$ are available $(10 \mathrm{~nm}$ full width at half maximum), whose transmitted mean photon energies $\langle h v\rangle$ are determined by the use of an absolutely calibrated high resolution spectrometer $(\langle h v\rangle=5.04-1.45 \mathrm{eV})$. Due to the fre- 


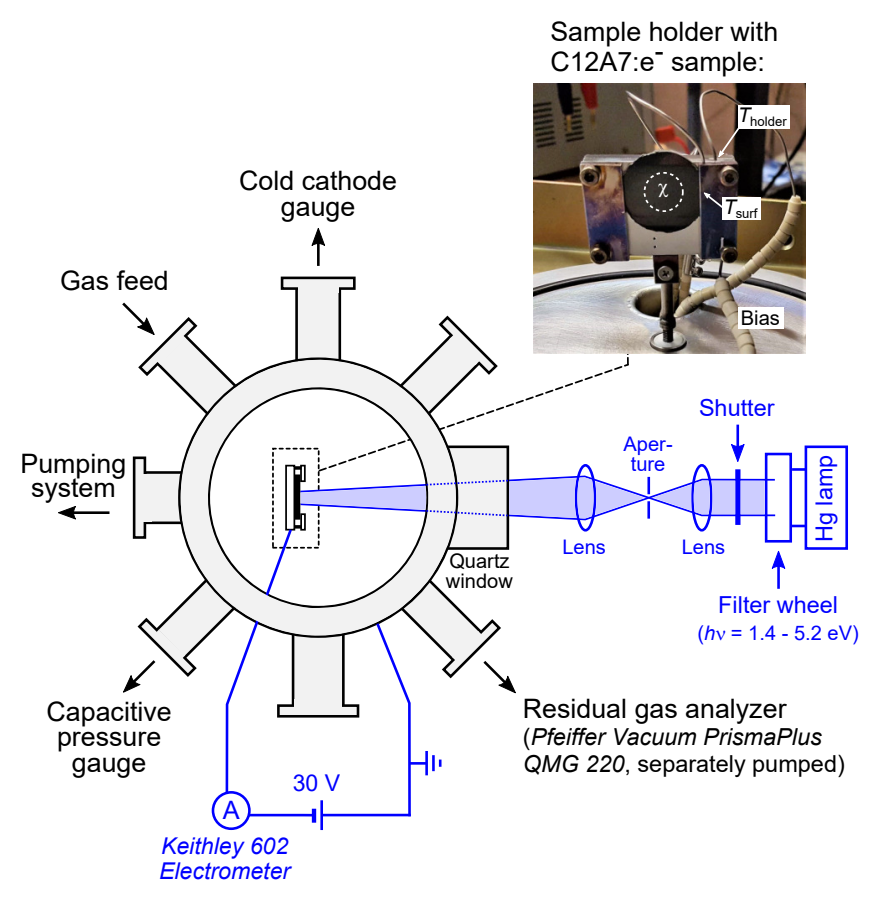

FIG. 1. Sketch of the ICP experiment from top view, with the work function diagnostic setup used. A picture of the mounted C12A7: $\mathrm{e}^{-}$ sample at the sample holder with the two installed thermocouples is shown in the top right corner. The illuminated spot on the sample surface for the work function measurement $(\chi)$ is indicated by the white dashed circle.

quency dependent emission intensity of the mercury lamp and the different transmissions of the applied interference filters, the irradiated power onto the sample surface is a function of the filter and is determined with a radiant power meter, giving values between $0.1-1 \mathrm{~mW}$. By biasing the electrically insulated sample holder with $-30 \mathrm{~V}$, the photoelectrically extracted electrons are drawn to the grounded vessel walls without space charge limitation and the respective current is monitored with a Keithley picoammeter. The dark current is measured by using a shutter in the optical path (in the range of $10^{-10} \mathrm{~A}$ ) and is subtracted from the current measurements for the determination of the energy resolved absolute photocurrents $I_{\mathrm{ph}}(\langle h v\rangle)$. Since during plasma operation a high current would be drawn by the application of $-30 \mathrm{~V}$ and would thus mask the photoelectrically emitted electrons, measurements of the work function are performed immediately after the plasma is switched off. The work function measurement itself takes about 2 min.

In a first approach, the photoelectric work function $\chi$ can roughly be identified via the threshold method, i.e., the transmitted photon energy of the filter for which a photoelectric response can still be detected gives an upper limit for the work function value. However, in order to determine the work function more accurately, extrapolation techniques should be used. A well-known and commonly used technique is the application of the semi-classical Fowler theory, ${ }^{40}$ after which the photoelectric current per unit incident light intensity, $I_{\mathrm{ph}, \mathrm{u}}$, is related to the photon energy near the work function threshold according to

$$
I_{\mathrm{ph}, \mathrm{u}}=C T^{2} f(\kappa)
$$

in which

$$
f(\kappa)=\int_{0}^{\infty} \ln [1+\exp (-y+\kappa)] \mathrm{d} y,
$$

$\kappa:=(h v-\chi) /\left(k_{\mathrm{B}} T\right), T$ is the absolute temperature of the irradiated surface and $k_{\mathrm{B}}$ is the Boltzmann constant. $C$ denotes a constant which is independent of $\chi, v$ and $T$ and depends on the optical absorption coefficient, photoelectric efficiency and Fermi level of the material. In the $T=0 \mathrm{~K}$ approximation, Eq. (1) predicts the quadratic dependence ${ }^{40}$

$$
I_{\mathrm{ph}, \mathrm{u}} \propto(h v-\chi)^{2}
$$

which has proven good applicability for clean metallic surfaces. ${ }^{41}$ Since the Fowler theory is, however, only valid for clean metallic surfaces, the more general power law

$$
I_{\mathrm{ph}, \mathrm{u}} \propto(h v-\chi)^{n}
$$

can be used for the extrapolation to zero photocurrent. The exponent $n$ depends on the excitation and escape mechanisms involved, and equals 2 in the case of the Fowler approximation (Eq. (3)). For adsorbate-covered or semiconducting surfaces, the $n$-parameter is typically in the range of $2.5-4 .^{42-46}$

\section{RESULTS AND DISCUSSION}

Before the measurements, the $\mathrm{C} 12 \mathrm{~A} 7: \mathrm{e}^{-}$sample was stored in atmospheric air. Energy-dispersive X-ray (EDX) spectroscopy was used for an elemental analysis of the sample, exhibiting mainly the presence of oxygen, aluminum and calcium, and some very small peaks related to carbon and silicon. After the installation of the sample in the vacuum chamber (see Fig. 1), the experiment is evacuated to a background pressure of about $5 \times 10^{-6}$ mbar. RGA monitoring shows that the main residual gases are water and nitrogen. The moderate vacuum level leads to a considerable gas flux on the order of $10^{19} \mathrm{~m}^{-2} \mathrm{~s}^{-1}$ onto the sample surface. Under these conditions and before any treatment of the electride, the measured surface work function is in the range of $\sim 5 \mathrm{eV}$. Such a high work function is most likely due to an adsorbate overlayer on the sample surface, resulting from the fabrication process and/or the air exposure. $5,37,47$

\section{A. Initial heating of the sample in vacuum}

In order to desorb the contaminant layer from the C12A7: $\mathrm{e}^{-}$surface, thermal annealing is applied in the first instance. The sample is heated in steps of $\Delta T=50 \mathrm{~K}$ from room temperature close to $T_{\text {surf }}=1000 \mathrm{~K}$, keeping the sample at each temperature step for about $15 \mathrm{~min}$. The measured temperature of the sample holder is about $15 \%$ higher than $T_{\text {surf }}$ and thus, a good thermal contact is provided. 
The photoelectric work function is measured at each temperature step. Since the PID controller of the heating device regulates the temperature stabilization via pulse-width modulation, the heating is switched off during the work function recording in order to avoid high noise in the current measurement via electromagnetic interference. When a surface temperature of about $475 \mathrm{~K}$ is reached, the measured photoelectric currents start to increase. For temperatures higher than $570 \mathrm{~K}$, the work function starts to decrease, and reaches a lowest value of $\sim 3.5 \mathrm{eV}$ for $T_{\text {surf }} \gtrsim 770 \mathrm{~K}$ (cf. Fig. 4). During the vacuum heat treatment, the RGA is used in the multiple ion detection mode to continuously monitor changes of the background gases with time. For the masses $18 \mathrm{u}\left(\mathrm{H}_{2} \mathrm{O}\right)$ and $44 \mathrm{u}\left(\mathrm{CO}_{2}\right)$, peaks occur each time the temperature is increased until $700 \mathrm{~K}$, and for the mass $28 \mathrm{u}\left(\mathrm{N}_{2} / \mathrm{CO}\right)$, peaks occur up to $1000 \mathrm{~K}$. The peaks in the RGA signals are accompanied with a short increase of the background pressure of up to $\Delta p=2.7 \times 10^{-5}$ mbar in maximum. The RGA signals in combination with the decrease of the surface work function indicate thermal desorption of impurities from the sample surface. Nonetheless, the lowest measured work function is still far above the literature value. This might be due to an insufficient cleaning and limited surface preparation under the given moderate vacuum conditions.

During the cooling down of the sample, the photoelectric efficiency and the work function show a degradation on the minute scale. When the sample reaches room temperature again, the surface work function is about $3.9 \mathrm{eV}$. Such a deterioration is most likely due to the readsorption of residual gases (mainly $\mathrm{H}_{2} \mathrm{O}$ ) at the vacuum-surface interface, which leads to changes of the electrostatic potential barriers and can promote an electron-deficient C12A7: $\mathrm{e}^{-}$surface layer. ${ }^{5}$ After about one week in vacuum, a work function of $\sim 4.9 \mathrm{eV}$ is obtained.

\section{B. Hydrogen plasma treatment}

Low pressure hydrogen discharges without active heating of the sample are ignited by feeding $10 \mathrm{~Pa} \mathrm{H}_{2}$ gas into the vacuum chamber and by applying $250 \mathrm{~W}$ RF power. The plasma environment with plasma parameters of $n_{\mathrm{e}} \sim 5 \times 10^{16} \mathrm{~m}^{-3}$ and $T_{\mathrm{e}} \sim 2 \mathrm{eV}^{39}$ leads to considerable particle fluxes onto the $\mathrm{C} 12 \mathrm{~A} 7: \mathrm{e}^{-}$sample surface: positive hydrogen ions (predominantly $\mathrm{H}_{3}^{+}$) bombard the surface with energies of up to $10 \mathrm{eV}$ and with a flux on the order of $10^{20} \mathrm{~m}^{-2} \mathrm{~s}^{-1}$ (sample at floating potential), the flux of neutral hydrogen atoms is on the order of $10^{22} \mathrm{~m}^{-2} \mathrm{~s}^{-1}\left(T_{\mathrm{H}} \approx 550 \mathrm{~K}\right)$, and photon fluxes of $10^{19}-10^{20} \mathrm{~m}^{-2} \mathrm{~s}^{-1}$ with energies of up to $15 \mathrm{eV}$ are created by resonant transitions of plasma particles. While the $\mathrm{H}_{2}$ gas without plasma ignition $\left(\Gamma_{\mathrm{H}_{2}} \sim 10^{24} \mathrm{~m}^{-2} \mathrm{~s}^{-1}\right)$ has no measurable influence on the work function, a one minute plasma pulse leads to an instant reduction of the work function from $3.9 \mathrm{eV}$ to $3.2 \mathrm{eV}$. Since the influx of the plasma particles heats the sample surface only by $+20 \mathrm{~K}$ during the pulse, thermal effects are negligible and the work function reduction can primarily be ascribed to the pure plasma-surface interaction. Without further plasma exposure, the work function increases

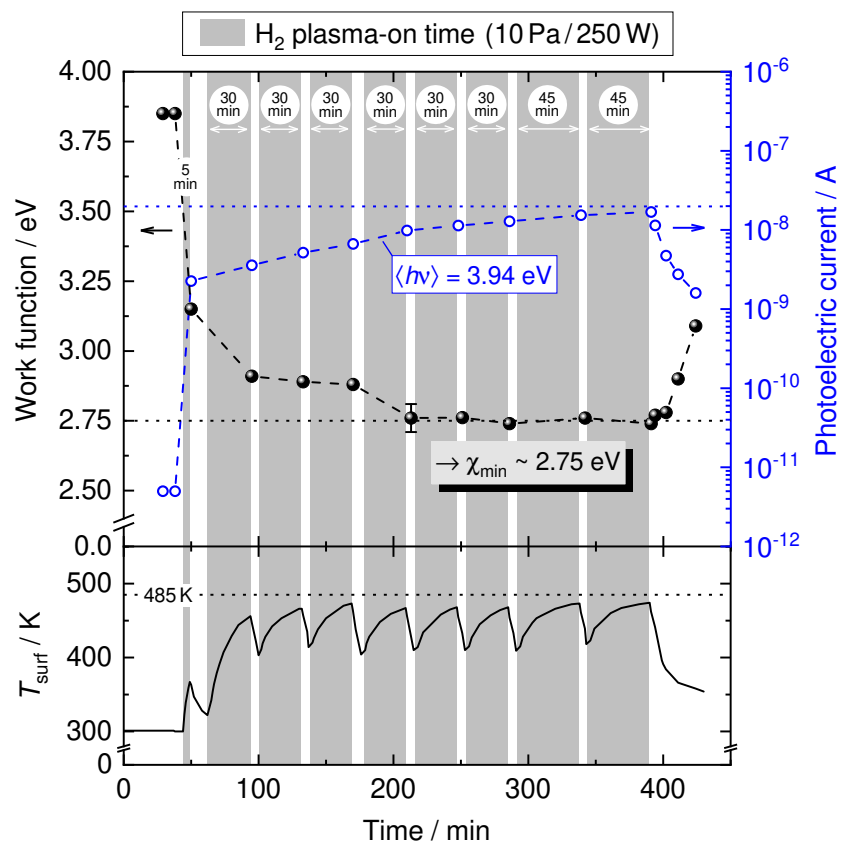

FIG. 2. Influence of hydrogen plasma pulses (10 Pa gas pressure, $250 \mathrm{~W}$ RF power) on the surface work function and the photoelectric response (exemplarily shown for $\langle h v\rangle=3.94 \mathrm{eV}$ ) of the C12A7: $\mathrm{e}^{-}$ sample. The impact of the impinging plasma species on the surface temperature is additionally depicted (no active heating applied). The steady-state values for further plasma-on time are represented by the horizontal dashed lines.

with a rate of about $+0.6 \mathrm{eV} / \mathrm{h}$ due to surface adsorption of residual gases and saturates at a value of $3.8-3.9 \mathrm{eV}$, i.e., within one hour the initial degraded work function is obtained again. By the subsequent application of a short hydrogen plasma pulse ( $\sim$ min), a work function of $3.2 \pm 0.1 \mathrm{eV}$ can be retrieved. Thus, the hydrogen plasma exposure is an efficient way to lower the surface work function of the C12A7: $\mathrm{e}^{-}$sample under the given experimental conditions, which is presumably due to an interplay of sputtering and chemically, ion- or photon-induced desorption processes of surface contaminants.

In Fig. 2, the influence of a long-term hydrogen plasma exposure on the temperature, photoelectric sensitivity and work function of the C12A7: $\mathrm{e}^{-}$surface is shown. As can be seen, the photoelectric response increases drastically after the first 5 min of plasma exposure (exemplarily plotted for an irradiated photon energy of $\langle h v\rangle=3.94 \mathrm{eV}$ ) and the evaluated surface work function drops by about $\Delta \chi=0.7 \mathrm{eV}$. By extending the plasma pulse length, the surface temperature increases considerably due to the impinging plasma particles and the work function is decreased further. After a plasma-on time of about two hours, the measured work function reaches a value of $\chi_{\min }=2.75 \pm 0.10 \mathrm{eV}$, which constitutes the lowest measured work function. While further plasma-on time does not lead to a further improvement of the work function, the photocurrents still increase slightly. The steady-state values obtained with further plasma exposure (not shown here) are depicted in Fig. 2.

Representative plots for the determination of the work func- 


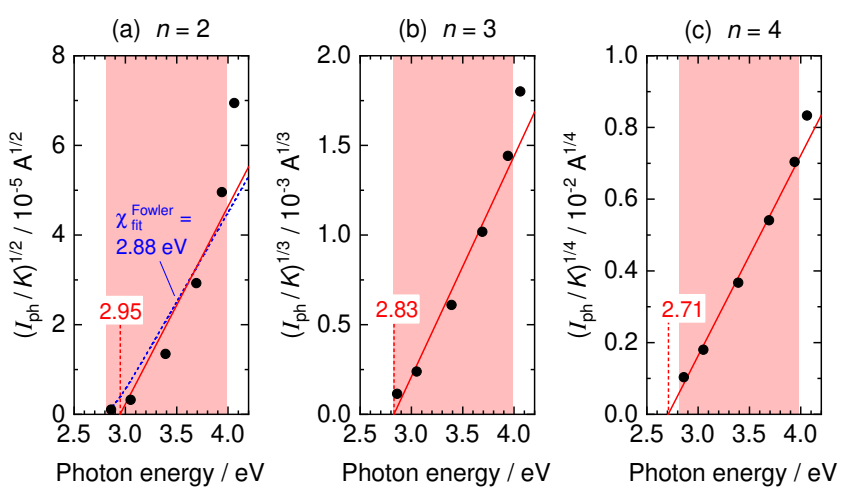

FIG. 3. Representative evaluation of the $\mathrm{C} 12 \mathrm{~A} 7: \mathrm{e}^{-}$surface work function after the application of hydrogen plasma $\left(T_{\text {surf }}=445 \mathrm{~K}\right)$ according to Eq. (4): the measured photoelectric yield data are extrapolated to zero photocurrent for (a) $n=2$, i.e., Fowler $T=0 \mathrm{~K}$ approximation, (b) $n=3$, and (c) $n=4$. Additionally, a Fowler fit according to Eq. (1) is shown in (a). The red shaded areas indicate the fitting range used.

tion according to Eq. (4) are depicted in Fig. 3. Before the evaluation, the measured absolute photocurrents $I_{\mathrm{ph}}(\langle h v\rangle)$ are divided by the respective relative intensities $K(\langle h v\rangle)$ of the light irradiation. Then, $\left(I_{\mathrm{ph}} / K\right)^{1 / n}$ is plotted as a function of the incident photon energy and the work function is determined by the $x$-intercept of a performed linear least-squares regression. In Fig. 3(a), the work function evaluation is illustrated for an $n$-parameter of 2 . As can be seen, the square root of the measured photocurrents in the range of roughly $1 \mathrm{eV}$ above threshold does not represent a straight line. Hence, also the plotted best-fit according to Eq. (1) does not match the progression of the data points well, since the Fowler theory predicts an approximately linear evolution of the square root of the photoelectric yield above threshold. In Fig. 3(b) and Fig. 3(c), the work function evaluation is performed for $n=3$ and $n=4$, respectively. In these representations, the linear fits are significantly improved: in the case of $n=3$, the linear regression predicts the measured data points with a coefficient of determination (COD) of $98.3 \%$ and yields an $x$-intercept of $2.83 \mathrm{eV}$, and in the case of $n=4$, the goodness of the fit is even enhanced, leading to a COD of $99.6 \%$ and an $x$-intercept of $2.71 \mathrm{eV}$. For the entirety of the present measurements, it turned out that an $n$-parameter of $n=3.5 \pm 0.5$ is most appropriate to fit the measured data. Using $n=3.5$ for the example in Fig. 3 gives a photoelectric threshold of $\chi_{\min }=2.75 \mathrm{eV}$.

The measured work function minimum is about $0.3-$ $0.4 \mathrm{eV}$ higher than the reported literature value for the $\mathrm{C} 12 \mathrm{~A} 7$ electride. The fact that the Fowler theory does not predict the photoelectric yield well in this work indicates that the C12A7: $\mathrm{e}^{-}$surface is not in a fully metallic state. This could imply a lowered Fermi level compared to anionic electron densities larger than $10^{21} \mathrm{~cm}^{-3}$ in the surface and subsurface cages and hence an elevated surface work function. ${ }^{6,7}$ Furthermore, it should be noted that the C12A7: $\mathrm{e}^{-}$surface is certainly not chemically pure under the given conditions here: the surface is exposed to a variety of particle fluxes arising from the plasma environment and the moderate vacuum conditions and hence, ad- and absorption processes of gas components might influence the work function as well.

As already mentioned, the $\mathrm{C} 12 \mathrm{~A} 7: \mathrm{e}^{-}$surface work function degrades with a rate of $+0.6 \mathrm{eV} / \mathrm{h}$ shortly after the plasma is switched off (cf. Fig. 2). After keeping the sample one day in vacuum, a plasma-on time of about one hour is needed to retrieve $\chi_{\min }$. In order to further remove impurities that might exist on the $\mathrm{C} 12 \mathrm{~A} 7: \mathrm{e}^{-}$surface, an argon plasma is applied for in total $100 \mathrm{~min}$ at a gas pressure of $10 \mathrm{~Pa}$ and an RF power of $200 \mathrm{~W}$. Moreover, the sample is biased with $-30 \mathrm{~V}$ to ground in order to reinforce the argon ion bombardment. During the argon plasma treatment, the sample temperature reaches $750 \mathrm{~K}$ in maximum. Afterwards, a significantly increased surface work function of $\sim 5 \mathrm{eV}$ is present, which can be ascribed to adsorbed argon atoms at the surface ${ }^{48}$ and/or a depleted occupation of the CCB as a result of the substantially higher flux of positive ions than electrons towards the C12A7: $\mathrm{e}^{-}$surface. With the subsequent application of hydrogen plasma without biasing the sample, the surface work function can be lowered again, but no beneficial effect of the argon plasma treatment and the associated additional heat treatment could be observed.

In total, the $\mathrm{C} 12 \mathrm{~A} 7: \mathrm{e}^{-}$surface exhibited a work function plateau of $2.75 \mathrm{eV}$ for a tested plasma-on time of $\sim 10 \mathrm{~h}$. Hence, the sample has proven to be stable under a long-term hydrogen plasma load with ion source relevant plasma parameters.

\section{Active heating of the sample during and after hydrogen plasma exposure}

Similar to the procedure described in section III A, the surface work function of the $\mathrm{C} 12 \mathrm{~A} 7: \mathrm{e}^{-}$sample is investigated during vacuum heat treatment after $\chi_{\min }=2.75 \mathrm{eV}$ has been reached and the surface has degraded to $\chi=3.9 \mathrm{eV}$ within one day in vacuum. The comparison between the heating before and after plasma treatment is depicted in Fig. 4. While the lowest achieved work function during the initial heating of the sample was $\sim 3.5 \mathrm{eV}$, the heating of the degraded surface after plasma treatment leads to a minimum of $2.9 \mathrm{eV}$ for temperatures in the range of $670-770 \mathrm{~K}$. This substantially lower work function is accompanied with a $2-3$ orders of magnitude higher photoelectric current drawn from the surface. Thus, the plasma exposure in between the two heating cycles led to an "activation" of the $\mathrm{C} 12 \mathrm{~A} 7: \mathrm{e}^{-}$surface in the sense that thermal treatment has become more efficient to provide a low work function surface state in the moderate vacuum environment.

During the long-term hydrogen plasma exposure, the plasma-induced increase of the sample temperature saturates at $\sim 485 \mathrm{~K}$. Heating the sample in vacuum up to this temperature leads to a work function of $3.25 \mathrm{eV}$. Hence, the about $0.5 \mathrm{eV}$ lower work function reached in the plasma environment can be directly attributed to the interaction of the impinging plasma species decoupled from possible thermal effects.

Interestingly, it is observed that the work function of the 


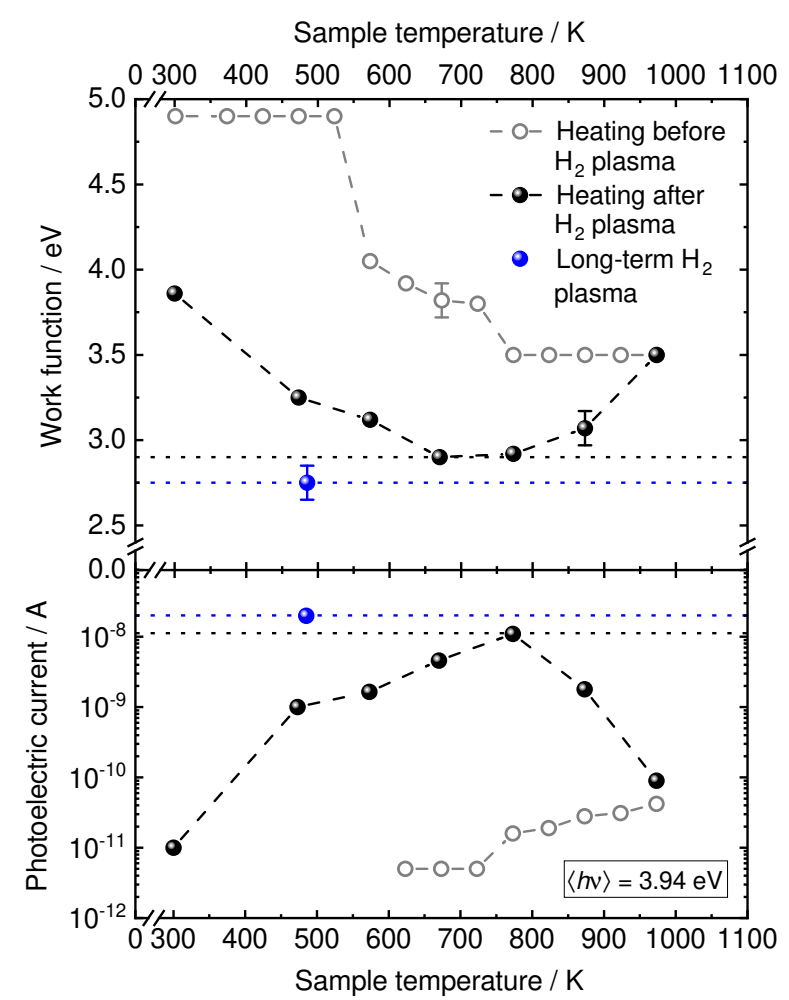

FIG. 4. Behavior of the C12A7: $\mathrm{e}^{-}$surface work function and photoelectric sensitivity during heat treatment in vacuum before and after the hydrogen plasma exposure (vacuum level of $5 \times 10^{-6} \mathrm{mbar}$ ). Additionally, the respective steady-state values attributed to long-term hydrogen plasma exposure are plotted for reference.

C12A7 electride increases and the photoelectric efficiency decreases when the sample temperature exceeds $\sim 770 \mathrm{~K}$. A similar behavior has been reported by Sasao et al. ${ }^{49}$ at a vacuum level of about two orders of magnitude lower than in this work. A plausible reason for such a behavior is that even under a very low partial pressure of $\mathrm{O}_{2}$ and/or $\mathrm{H}_{2} \mathrm{O}$, the anionic electrons in the cages can be replaced by $\mathrm{O}^{2-}$ and/or $\mathrm{OH}^{-}$ during annealing and can produce insulation regions on the C12A7: $\mathrm{e}^{-}$surface. ${ }^{13}$ By the application of hydrogen plasma pulses after the cooling down of the sample to $T_{\text {surf }}=330 \mathrm{~K}$, the low work function of $2.75 \mathrm{eV}$ can be recovered within a plasma-on time of about $50 \mathrm{~min}$. Hence, a possible exchange of anionic electrons is suggested to be reversible in the plasma environment.

Further, the C12A7: $\mathrm{e}^{-}$sample is actively heated in the hydrogen plasma environment in order to promote a synergistic benefit of plasma and thermal effects. The sample temperature is increased to $\sim 670 \mathrm{~K}$ during long-term plasma exposure, which is the temperature with which the work function minimum is reached during the vacuum heat treatment. Compared to the measurements without active heating during plasma, however, no beneficial effect is observed.

\section{Ex situ analysis}

After the completion of the experimental campaign, the $\mathrm{C} 12 \mathrm{~A} 7: \mathrm{e}^{-}$sample is removed from the vacuum chamber and kept in ambient atmosphere. Pictures of the sample before and after the experimental campaign are provided in Fig. 5, where "front side" denotes the surface which was exposed to plasma and "rear side" the surface which was directed towards the sample holder. Before the campaign, both sides of the sample exhibit a homogeneous and black surface, with some small cracks in the center of the rear side resulting from the fabrication process. After the campaign, no changes of the front side surface texture can be detected by eye, optical microscopy, scanning electron microscopy (SEM), and EDX spectroscopy. Thus, it can be concluded that the $\mathrm{C} 12 \mathrm{~A} 7: \mathrm{e}^{-}$surface is not susceptible to plasma-induced erosion.

The surface which was directed towards the stainless steel sample holder shows, however, a considerable color change from black to yellowish-brown. Only the regions on the rear side without direct contact to the sample holder could preserve the black coloration, which is the upper part (cf. Fig. 1) and positions where the holder provides holes for outgassing and diagnostic purposes. The color change indicates a degeneration of the C12A7: $\mathrm{e}^{-}$surface state, which might be due to an extensive heat treatment in direct contact with the stainless steel $\left(T_{\text {holder }}=1080 \mathrm{~K}\right.$ in maximum) and/or due to adsorbates from ambient air from before the installation at the sample holder. Moreover, a horizontal crack across the sample developed (clearly visible under the optical microscope) and some small pieces at the positions where the sample was clamped to the sample holder flaked off. These mechanical damages are probably the result of thermal stress at unfavorably high temperatures of $\sim 1000 \mathrm{~K}$. Since such high temperatures have also shown an adverse effect on the work function performance, it is recommended to not exceed a temperature range of $700-800 \mathrm{~K}$.

\section{CONCLUSIONS}

In this study, a polycrystalline $\mathrm{C} 12 \mathrm{~A} 7: \mathrm{e}^{-}$ceramic with an incorporated electron carrier concentration of $1.0 \times 10^{21} \mathrm{~cm}^{-3}$ was tested in view of a potential application as converter surface material in negative hydrogen ion sources. The electride has shown good resilience to long-term hydrogen plasma exposure and exhibits a stable low work function plateau of $2.75 \pm 0.10 \mathrm{eV}$. The conditioning procedure for reaching the measured work function minimum included vacuum annealing close to $1000 \mathrm{~K}$ and a plasma-on time on the hour scale. The latter implies that under the given experimental conditions, the optimum performance of a $\mathrm{C} 12 \mathrm{~A} 7: \mathrm{e}^{-}$converter surface for negative hydrogen ions is only reached after keeping the surface for $\gtrsim 1 \mathrm{~h}$ continuously in the hydrogen plasma environment. For negative ion sources operating in pulsed mode, however, (i.e., application of short plasma pulses with a high repetition rate) the conditioning and performance of C12A7: $\mathrm{e}^{-}$should be investigated dedicatedly.

After the plasma is switched off, the drastic decrease of the 

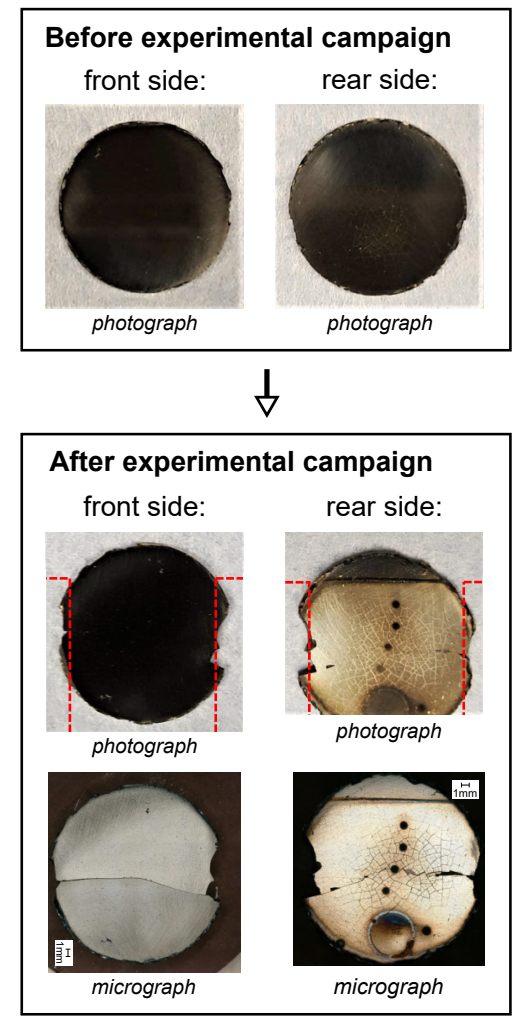

FIG. 5. Pictures of the C12A7: $\mathrm{e}^{-}$sample (ordinary photographs and optical micrographs) before and after the conducted experiments in the plasma chamber, with front side denoting the surface which was directed towards the plasma and rear side the surface which was directed towards the sample holder. Some pieces at the positions where the sample was clamped to the holder flaked off (clamp positions indicated by red dashed lines) and a horizontal crack across the sample appeared. The small cracks on the rear side originate from the fabrication process.

photoelectric efficiency accompanied by the increase of the surface work function with a rate of $+0.6 \mathrm{eV} / \mathrm{h}$ shows that the electride is very sensitive to residual gases, which can adsorb unhindered on the surface in the absence of impinging plasma species (vacuum level of $\sim 10^{-6}$ mbar). The work function degradation results in an elevated surface work function of $3.9 \mathrm{eV}$ on the next day. By the application of hydrogen plasma pulses, a work function of $2.75 \mathrm{eV}$ can be retrieved within a plasma-on time of minutes to hours, depending on the degradation state. Thus, the surface degradation is effectively counteracted and reversed by hydrogen plasma exposure.

Heating the C12A7: $\mathrm{e}^{-}$sample with a degraded surface work function after plasma activation in vacuum reduces the work function to $3.25 \mathrm{eV}$ at $480 \mathrm{~K}$ and $2.9 \mathrm{eV}$ in a temperature range of $670-770 \mathrm{~K}$. Since the plasma-surface interaction in steady-state leads to a surface temperature of about $480 \mathrm{~K}$ and a work function of $2.75 \mathrm{eV}$, it can be concluded that the hydrogen plasma environment has a beneficial impact on the C12A7: $\mathrm{e}^{-}$surface besides thermal effects. Exceeding a temperature of $\sim 770 \mathrm{~K}$ results in a disadvantageous effect regarding the photoemissive properties and surface work func- tion of the electride.

The reached work function minimum with the tested C12A7: $\mathrm{e}^{-}$surface is about $0.35 \mathrm{eV}$ higher than the reported value of $2.4 \mathrm{eV}$ for the chemically pure bulk under UHV conditions. It should be checked in a next step whether an increase of the electron carrier concentration beyond $1.0 \times$ $10^{21} \mathrm{~cm}^{-3}$ leads to a further enhancement towards $2.4 \mathrm{eV}$. The currently reached work function minimum is relatively far above $2 \mathrm{eV}$, which is achieved with the state-of-the-art technique of in situ caesiation of metallic surfaces. Consequently, the $\mathrm{H}^{-}$conversion yield via direct electron transfer from the C12A7: $\mathrm{e}^{-}$surface is expected to be much smaller compared to a Cs loaded surface, and subsequent campaigns must show whether the higher work function can be overcompensated by other $\mathrm{H}^{-}$production channels and/or a reduced complexity of handling due to its air-stability and chemical inertness.

\section{ACKNOWLEDGMENTS}

This work has been carried out within the framework of the EUROfusion Consortium and has received funding from the Euratom research and training programme $2014-2018$ and 2019 - 2020 under grant agreement No 633053. The views and opinions expressed herein do not necessarily reflect those of the European Commission.

\section{DATA AVAILABILITY}

The data that support the findings of this study are available from the corresponding author upon reasonable request.

${ }^{1}$ S. Matsuishi, Y. Toda, M. Miyakawa, K. Hayashi, T. Kamiya, M. Hirano, I. Tanaka, and H. Hosono, "High-Density Electron Anions in a Nanoporous Single Crystal: $\left[\mathrm{Ca}_{24} \mathrm{Al}_{28} \mathrm{O}_{64}\right]^{4+}\left(4 e^{-}\right)$," Science 301, 626-629 (2003).

${ }^{2} \mathrm{H}$. Hosono, "Functioning of traditional ceramics $12 \mathrm{CaO} \cdot 7 \mathrm{Al}_{2} \mathrm{O}_{3}$ utilizing built-in nano-porous structure," Science and Technology of Advanced Materials 5, 409-416 (2004).

${ }^{3}$ P. V. Sushko, A. L. Shluger, K. Hayashi, M. Hirano, and H. Hosono, "Electron Localization and a Confined Electron Gas in Nanoporous Inorganic Electrides," Physical Review Letters 91, 126401 (2003).

${ }^{4}$ P. V. Sushko, A. L. Shluger, M. Hirano, and H. Hosono, "From Insulator to Electride: A Theoretical Model of Nanoporous Oxide $12 \mathrm{CaO} \cdot 7 \mathrm{Al}_{2} \mathrm{O}_{3}$," Journal of the American Chemical Society 129, 942-951 (2007).

${ }^{5}$ Y. Toda, H. Yanagi, E. Ikenaga, J. J. Kim, M. Kobata, S. Ueda, T. Kamiya, M. Hirano, K. Kobayashi, and H. Hosono, "Work Function of a RoomTemperature, Stable Electride $\left.\mathrm{Ca}_{24} \mathrm{Al}_{28} \mathrm{O}_{64}\right]^{4+}\left(\mathrm{e}^{-}\right)_{4}$," Advanced Materials 19, 3564-3569 (2007).

${ }^{6}$ S. W. Kim, S. Matsuishi, T. Nomura, Y. Kubota, M. Takata, K. Hayashi, T. Kamiya, M. Hirano, and H. Hosono, "Metallic State in a LimeAlumina Compound with Nanoporous Structure," Nano Letters 7, 11381143 (2007).

${ }^{7}$ S. W. Kim and H. Hosono, "Synthesis and properties of $12 \mathrm{CaO} \cdot 7 \mathrm{Al}_{2} \mathrm{O}_{3}$ electride: review of single crystal and thin film growth," Philosophical Magazine 92, 2596-2628 (2012).

${ }^{8}$ S. W. Kim, M. Miyakawa, K. Hayashi, T. Sakai, M. Hirano, and H. Hosono, "Simple and efficient fabrication of room temperature stable electride: Melt-solidification and glass ceramics," Journal of the American Chemical Society 127, 1370-1371 (2005).

${ }^{9}$ F. Li, X. Zhang, H. Liu, J. Zhao, Y. Xiao, Q. Feng, and J. Zhang, "In situ synthesis of $\left[\mathrm{Ca}_{24} \mathrm{Al}_{28} \mathrm{O}_{64}\right]^{4+}\left(4 \mathrm{e}^{-}\right)$electride ceramic from C12A7 + C3A mixture precursor,' Journal of the American Ceramic Society 102, 884-888 (2019). 
${ }^{10} \mathrm{Y}$. Xiao, X. Zhang, and J. Zhang, " $\left[\mathrm{Ca}_{24} \mathrm{Al}_{28} \mathrm{O}_{64}\right]^{4+}\left(4 \mathrm{e}^{-}\right)$electrides ceramic with high-electron concentration rapidly fabricated by spark plasma sintering of $\mathrm{Ca}, \mathrm{Al}$ organic powder precursor in spark plasma sintering," Ceramics International 46, 27742-27749 (2020).

${ }^{11}$ S.-W. Kim, S. Matsuishi, M. Miyakawa, K. Hayashi, M. Hirano, and $\mathrm{H}$. Hosono, "Fabrication of room temperature-stable $12 \mathrm{CaO} \cdot 7 \mathrm{Al}_{2} \mathrm{O}_{3}$ electride: a review," Journal of Materials Science: Materials in Electronics 18, 5-14 (2007).

${ }^{12} \mathrm{~F}$. Li, X. Zhang, and $\mathrm{H}$. Liu, "Insights into the direct formation of $\left[\mathrm{Ca}_{24} \mathrm{Al}_{28} \mathrm{O}_{64}\right]^{4+}\left(4 \mathrm{e}^{-}\right)$and its electrical characterization," Journal of the American Ceramic Society 103, 35-42 (2020).

${ }^{13}$ Y. Toda, Y. Kubota, M. Hirano, H. Hirayama, and H. Hosono, "Surface of room-temperature-stable electride $\left[\mathrm{Ca}_{24} \mathrm{Al}_{28} \mathrm{O}_{64}\right]^{4+}\left(\mathrm{e}^{-}\right)_{4}$ : Preparation and its characterization by atomic-resolution scanning tunneling microscopy," ACS Nano 5, 1907-1914 (2011).

${ }^{14}$ H. Hosono, S.-W. Kim, S. Matsuishi, S. Tanaka, A. Miyake, T. Kagayama, and K. Shimizu, "Superconductivity in room-temperature stable electride and high-pressure phases of alkali metals," Philosophical Transactions of the Royal Society A: Mathematical, Physical and Engineering Sciences 373, 20140450 (2015)

${ }^{15}$ K.-B. Kim, M. Kikuchi, M. Miyakawa, H. Yanagi, T. Kamiya, M. Hirano, and H. Hosono, "Photoelectron spectroscopic study of C12A7: $\mathrm{e}^{-}$and $\mathrm{Alq}_{3}$ interface: The formation of a low electron-injection barrier," The Journal of Physical Chemistry C 111, 8403-8406 (2007).

${ }^{16}$ H. Yanagi, T. Kuroda, K.-B. Kim, Y. Toda, T. Kamiya, and H. Hosono, "Electron injection barriers between air-stable electride with low work function, $\mathrm{C} 12 \mathrm{~A} 7: \mathrm{e}^{-}$, and pentacene, $\mathrm{C}_{60}$ and copper phthalocyanine," Journal of Materials Chemistry 22, 4278-4281 (2012).

${ }^{17}$ D. Kuwahara, T. Kasuya, M. Sasao, and M. Wada, "Study of negative hydrogen ion production at low work function material surface using a hydrogen atom source," AIP Conference Proceedings 1869, 020009 (2017).

${ }^{18}$ W. Zou, K. Khan, X. Zhao, C. Zhu, J. Huang, J. Li, Y. Yang, and W. Song, "Direct fabrication of C12A7 electride target and room temperature deposition of thin films with low work function," Materials Research Express 4, 036408 (2017).

${ }^{19}$ Y. Toda, S. W. Kim, K. Hayashi, M. Hirano, T. Kamiya, H. Hosono, T. Haraguchi, and H. Yasuda, "Intense thermal field electron emission from room-temperature stable electride," Applied Physics Letters 87, 254103 (2005).

${ }^{20}$ D. R. Lev, I. G. Mikellides, D. Pedrini, D. M. Goebel, B. A. Jorns, and M. S. McDonald, "Recent progress in research and development of hollow cathodes for electric propulsion," Reviews of Modern Plasma Physics 3, 6 (2019).

${ }^{21}$ H. Buchammagari, Y. Toda, M. Hirano, H. Hosono, D. Takeuchi, and K. Osakada, "Room temperature-stable electride as a synthetic organic reagent: Application to pinacol coupling reaction in aqueous media," Organic Letters 9, 4287-4289 (2007).

${ }^{22}$ M. Kitano, Y. Inoue, Y. Yamazaki, F. Hayashi, S. Kanbara, S. Matsuishi, T. Yokoyama, S.-W. Kim, M. Hara, and H. Hosono, "Ammonia synthesis using a stable electride as an electron donor and reversible hydrogen store," Nature chemistry 4, 934-940 (2012).

${ }^{23}$ M. Hara, M. Kitano, and H. Hosono, "Ru-loaded C12A7: ${ }^{-}$electride as a catalyst for ammonia synthesis," ACS Catalysis 7, 2313-2324 (2017).

${ }^{24}$ M. Sasao, R. Moussaoui, D. Kogut, J. Ellis, G. Cartry, M. Wada, K. Tsumori, and H. Hosono, "Negative-hydrogen-ion production from a nanoporous $12 \mathrm{CaO} \cdot 7 \mathrm{Al}_{2} \mathrm{O}_{3}$ electride surface," Applied Physics Express 11, 066201 (2018).

${ }^{25}$ U. Fantz and J. Lettry, "Focus on sources of negatively charged ions," New Journal of Physics 20, 060201 (2018).

${ }^{26}$ M. Bacal and M. Wada, "Negative hydrogen ion production mechanisms," Applied Physics Reviews 2, 021305 (2015).

${ }^{27}$ T. Morishita, M. Kashiwagi, M. Hanada, Y. Okumura, K. Watanabe, A. Hatayama, and M. Ogasawara, "Mechanism of negative ion production in a cesium seeded ion source," Japanese Journal of Applied Physics 40, 4709-4714 (2001).

${ }^{28}$ R. Friedl and U. Fantz, "Influence of $\mathrm{H}_{2}$ and $\mathrm{D}_{2}$ plasmas on the work function of caesiated materials," Journal of Applied Physics 122, 083304 (2017).
${ }^{29}$ S. Cristofaro, R. Friedl, and U. Fantz, "Correlation of Cs flux and work function of a converter surface during long plasma exposure for negative ion sources in view of ITER," Plasma Research Express 2, 035009 (2020). ${ }^{30}$ G. Cartry, D. Kogut, K. Achkasov, J. M. Layet, T. Farley, A. Gicquel, J. Achard, O. Brinza, T. Bieber, H. Khemliche, P. Roncin, and A. Simonin, "Alternative solutions to caesium in negative-ion sources: a study of negative-ion surface production on diamond in $\mathrm{H}_{2} / \mathrm{D}_{2}$ plasmas," New Journal of Physics 19, 025010 (2017).

${ }^{31}$ D. Kogut, R. Moussaoui, N. Ning, J. B. Faure, J. M. Layet, T. Farley, J. Achard, A. Gicquel, and G. Cartry, "Impact of positive ion energy on carbon-surface production of negative ions in deuterium plasmas," Journal of Physics D: Applied Physics 52, 435201 (2019).

${ }^{32}$ L. Schiesko, G. Cartry, C. Hopf, T. Höschen, G. Meisl, O. Encke, B. Heinemann, K. Achkasov, P. Amsalem, and U. Fantz, "First experiments with cs doped mo as surface converter for negative hydrogen ion sources," Journal of Applied Physics 118, 073303 (2015).

${ }^{33}$ Y. I. Belchenko, G. I. Kuznetsov, and E. A. Grigoryev, "Hydrogen negative ion source with $\mathrm{LaB}_{6}$ inserts," Review of Scientific Instruments 71, 10791081 (2000).

${ }^{34}$ U. Kurutz, R. Friedl, and U. Fantz, "Investigations on cs-free alternatives for negative ion formation in a low pressure hydrogen discharge at ion source relevant parameters," Plasma Physics and Controlled Fusion 59, 075008 (2017).

${ }^{35}$ R. Friedl, S. Cristofaro, and U. Fantz, "Work function of Cs-free materials for enhanced $\mathrm{H}^{-}$surface production," AIP Conference Proceedings 2011, 050009 (2018).

${ }^{36}$ U. Fantz, C. Hopf, R. Friedl, S. Cristofaro, B. Heinemann, S. Lishev, and A. Mimo, "Technology developments for a beam source of an NNBI system for DEMO," Fusion Engineering and Design 136, 340-344 (2018).

${ }^{37}$ K. Waetzig, A. Rost, J. Schilm, M. Tajmar, and A. Michaelis, "Contacting Methods for C12A7 Electride Ceramic," Journal of Ceramic Science and Technology 11, 11-16 (2020).

${ }^{38} \mathrm{R}$. Friedl and U. Fantz, "Influence of cesium on the plasma parameters in front of the plasma grid in sources for negative hydrogen ions," AIP Conference Proceedings 1515, 255-262 (2013).

${ }^{39}$ R. Friedl and U. Fantz, "Fundamental studies on the Cs dynamics under ion source conditions," Review of Scientific Instruments 85, 02B109 (2014).

${ }^{40}$ R. H. Fowler, "The Analysis of Photoelectric Sensitivity Curves for Clean Metals at Various Temperatures," Physical Review 38, 45-56 (1931).

${ }^{41}$ D. E. Eastman, "Photoelectric Work Functions of Transition, Rare-Earth, and Noble Metals," Physical Review B 2, 1-2 (1970).

${ }^{42}$ G. W. Gobeli and F. G. Allen, "Direct and Indirect Excitation Processes in Photoelectric Emission from Silicon," Physical Review 127, 141-149 (1962).

${ }^{43}$ T. E. Fischer, "Determination of semiconductor surface properties by means of photoelectric emission," Surface Science 13, 30-51 (1969).

${ }^{44}$ J. A. Assimos and D. Trivich, "The photoelectric threshold, work function, and surface barrier potential of single-crystal cuprous oxide," physica status solidi (a) 26, 477-488 (1974).

${ }^{45}$ P. Lange, J. K. Sass, R. Unwin, and D. M. Tench, "Improved analysis of the power law in photoemission yield spectroscopy," Journal of Electroanalytical Chemistry and Interfacial Electrochemistry 122, 387-391 (1981).

${ }^{46}$ P. Lange, D. Grider, H. Neff, J. K. Sass, and R. Unwin, "Limitations of the Fowler method in photoelectric work function determination: Oxygen on magnesium single crystal surfaces," Surface Science Letters 118, L257L262 (1982).

${ }^{47}$ S. Matsuishi, S. W. Kim, T. Kamiya, M. Hirano, and H. Hosono, "Localized and delocalized electrons in room-temperature stable electride $\left[\mathrm{Ca}_{24} \mathrm{Al}_{28} \mathrm{O}_{64}\right]^{4+}\left(\mathrm{O}^{2-}\right)_{2-x}\left(e^{-}\right)_{2 x}$ : Analysis of optical reflectance spectra," The Journal of Physical Chemistry C 112, 4753-4760 (2008).

${ }^{48}$ O. Y. Kolesnychenko, O. I. Shklyarevskii, and H. van Kempen, "Anomalous increase of the work function in metals due to adsorbed helium," Physica B: Condensed Matter 284-288, 1257-1258 (2000).

${ }^{49}$ M. Sasao, K. Tanemura, M. Wada, and K. Tsumori, "Negative ion formation from a low-work-function nanoporous inorganic electride surface," AIP Conference Proceedings 1869, 020005 (2017). 